\title{
Measurement of Vehicle Parking Deviation Angle Based on Machine Vision
}

\author{
Peijiang Chen ${ }^{1,2}$ and Yongjun Min ${ }^{1, *}$ \\ ${ }^{1}$ College of Mechanical and Electronic Engineering, Nanjing Forestry University, Jiangsu, 210037, China \\ ${ }^{2}$ School of Automobile, Linyi University, Shandong, 276000, China
}

\begin{abstract}
In order to improve the detection accuracy and increase the automation level of some items in vehicle detection, a method of computing vehicle parking deviation angle based on machine vision was proposed. The feature points of left and right vehicle images were detected by SIFT (Scale Invariant Feature Transform) algorithm. Euclidean distance was used as the similarity measurement factor, the feature points matching algorithm based on bidirectional searching was put forward. The matched points of left image was divided into two sets, on the left and right half planes. The feature vectors on right half was corrected symmetrically, and the symmetric points were detected. By camera calibration, the threedimensional coordinates of midpoints were obtained and the vehicle longitudinal axis was received, finally the vehicle parking deviation angle was analyzed. The novel contribution of this method was that the measurement is based on feature points detection and symmetric feature points matching. The experiment results showed that this method effectively detected the parking deviation angle, which could be applied in vehicle detection and provided reference to correct the measurement data of vehicle testing.
\end{abstract}

Keywords: Bidirectional search, camera calibration, parking deviation angle, sift, symmetric feature.

\section{INTRODUCTION}

With the advancement of automotive technology and the increase of car ownership, people's daily life has been inseparable from the car, in order to ensure the safety of persons and vehicles, vehicle performance detection and fault diagnosis are essential and crucial. Accordingly, the technologies of vehicle detection and maintenance are constantly improved, all kinds of advanced instruments, equipment and artificial intelligence methods [1-5] have emerged, and the projects of vehicle detection also continuously enrich.

Vehicle parking deviation angle can be seen as the angle between the actual parking position and the ideal site in vehicle detection field. The deviation angle is used in some vehicle detection items. For example, the vehicle longitudinal axis should be vertical with the rail of the headlamp tester in headlamp detection or the test results are not satisfactory and bring measurement errors. This error can be corrected by detecting the vehicle parking deviation angle. This data correction can improve the accuracy of headlight detection.

The detection of vehicles parking deviation angle has mainly three ways: method based on digital image processing, method based on machine vision and method based on laser ranging.

When studying the technologies of automobile headlamp detection, in order to reduce the testing error, Zhu [6], Zhu

*Address correspondence to this author at the College of Mechanical and Electronic Engineering, Nanjing Forestry University, Jiangsu, 210037, China; Tel: +086-025-85427185; E-mail: yjmin@njfu.edu.cn
[7], Min et al. [8] early proposed a method of analyzing the angle of vehicle parking deviation which was used to correct the measured data of headlamp tester. The main idea is to use image segmentation technology [9] to analyze the vehicle overhead image, apply approximation method to calculate the vehicle longitudinal axis and obtain the vehicle parking deviation angle. Yun et al. [10] also adapted the image processing method to acquire the vehicle longitudinal axis deviation. Wang [11] used the detected midpoints of the tail and rear of car to get the centerline and then the deviation angle. Chen and Min [12] achieved the calculation of vehicle parking deviation angle based on camera calibration technologies, which took the deflection between the image and tested field into account, making the detection results more suitable with the actual situation. The detection methods based on image processing require little hardware and has good real time performance. But the drawback of this method is that the detection precision largely depends on the image processing technology, particularly the image segmentation technology, it can seriously affect the detection results.

Lin [13] introduced the stereo vision technology to the detection of axis position deviation, the automobile was left on the automobile straightening apparatus, collecting two images of an automobile body, extracted the boundary lines within certain slopes and lengths, got their centerlines, finally obtained the vehicle parking deviation angle. This method has good accuracy, but need special straightening apparatus which is expensive and the performance of real time is poor. 
$\mathrm{Wu}$ [14] used laser ranging technology to detect the automobile body parking error. Laser rangefinder was set in front of the tested automobile and the reflective surfaces were installed above the car. According to the distance between two rangefinders and the distances between the reflective surfaces and the headlamp testers, the direction of vehicle parking deviation angle was detected. Since the reflective surfaces should be installed, adjusted and demounted in each testing process, it is very inconvenient and has bad real time property, and the measurement accuracy is not easy ensured. Hou [15], Zhang et al. [16, 17], Shan et al. [18] designed a device for headlamp detection error correction. The contour curve of the rear tire was received through laser rangefinder and the tire profile was identified, placing angle of rear axle was obtained, then the skew angle of driving direction was determined. The method makes the driving direction as reference and it has good practical results. But, the wheel inclination angle is not entirely in accordance with the vehicle parking deviation angle, affecting the automation degree and measurement accuracy.

Through the above analysis, the three commonly used methods have their own advantages and disadvantages, each method cannot solve the problem perfectly. The measurement method based on machine vision has many merits, such as high efficiency, appropriate precision, simple system structure, low cost, and so on. It is very suitable for online, non-contact measuring and controlling system. In addition, the measuring system in this study does not use the automobile straightening apparatus, then, the measurement process can realize automatic operation.

Aiming at the demand for vehicle detection, a vehicle parking deviation angle detection system based on machine vision technology is built. Two images of the same vehicle are captured by binocular stereo camera. The feature points of images are detected by SIFT algorithm, the features are measured by Euclidean distance, and feature point matching is realized based on bidirectional searching. Symmetric pairs of feature points on vehicle body are detected according to symmetric feature vectors, the coordinates of midpoints are received in the world coordinate system, finally the vehicle parking deviation angle is analyzed. The novel contribution of this study is that the measuring method is based on feature points detection and symmetric feature points matching. This method can establish foundation for the next application in vehicle detection and it has good practical value and application foreground.

\section{DESIGN OF VEHICLE PARKING DEVIATION ANGLE DETECTION SYSTEM BASED ON MA- CHINE VISION}

\subsection{Binocular Stereo Vision System}

It is a method of acquiring three dimensional geometrical information of an object by two images [19]. The theory is based on parallax principle [20], it gets two images from different locations of the same thing, calculates the comparative poison deviation of the corresponding points to acquire the three dimensional information [21].
The system fundamental is given, as shown in Fig. (1). Oxyz is world coordinates system, $O_{l} x_{l} y_{l} z_{l}$ and $O_{r} x_{r} y_{r} z_{r}$ are coordinates systems for left camera and right camera respectively, $o_{l} u_{l} v_{l}$ and $o_{r} u_{r} v_{r}$ are coordinates systems for left image and right image respectively. $a(x, y, z)$ is a space point, which projection points in left and right image coordinates systems are respectively $a_{l}\left(u_{l}, v_{l}\right)$ and $a_{r}\left(u_{r}, v_{r}\right)$.

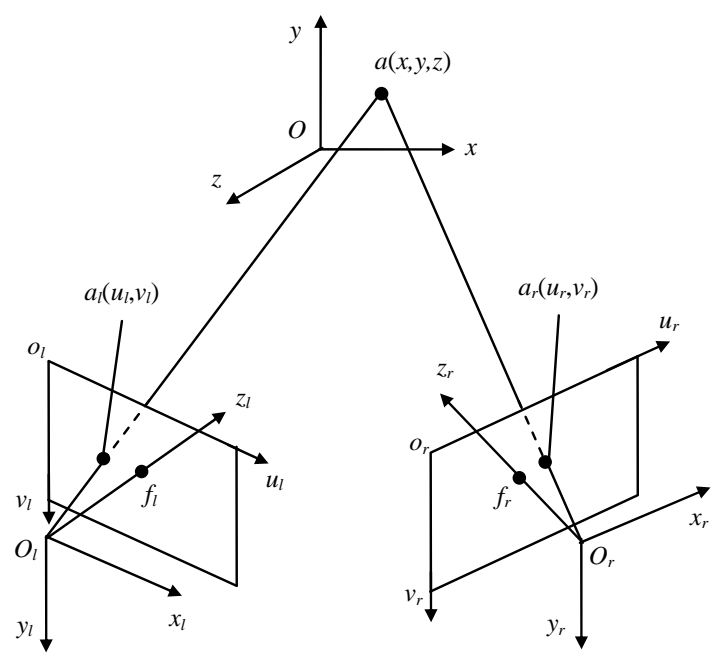

Fig. (1). The basic principle of binocular stereo vision.

\subsection{Overall Design Plan of Vehicle Parking Deviation Angle Detection System}

The deviation angle of vehicle parking is the deviation between the vehicle's centerline and desired parking position. The vehicle's longitudinal axis is the intersection line of symmetric plane of vehicle and testing ground plane. According to the characteristics of vehicle parking deviation angle detection, based on machine vision, a method of detecting deviation angle is proposed in this study.

In the simplest case, as long as two symmetric points are found, connecting them into a line and drawing the vertical plane of this line and then the vehicle longitudinal axis can be received. Or, more than two pairs of symmetric points are obtained, connecting all midpoints of symmetric point pairs to form the centerline. On the basis of longitudinal axis, it is easy to get the vehicle deviation angle. Thus, the key task of this study is to detect the longitudinal axis of vehicle.

The system design principle is as follows. On the premise of camera calibration, firstly the feature points are extracted and matched in two captured vehicle images, and the symmetric points of matched feature pointes are detected in left image, the three-dimensional coordinates of feature points are calculated in world coordinate system, then the vehicle longitudinal axis is obtained, finally the vehicle parking deviation angle is gotten.

Based on the technologies of binocular stereo vision, the architecture of vehicle parking deviation angle detection system is designed as shown in Fig. (2). 


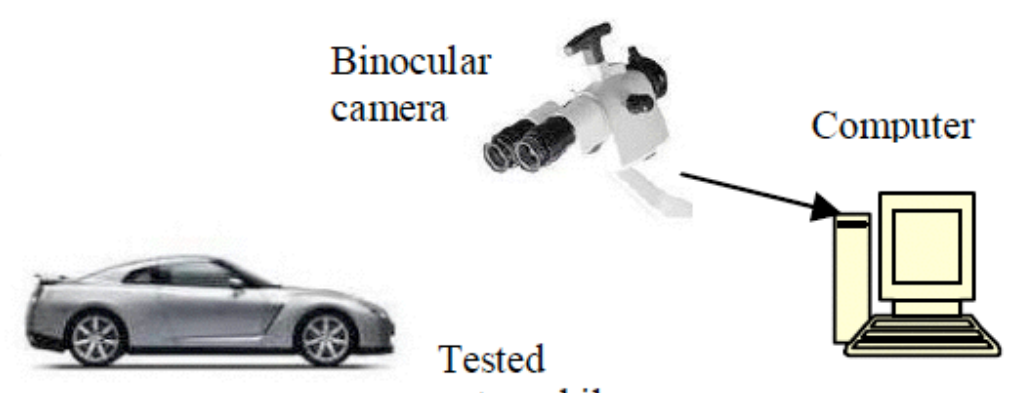

automobile

Fig. (2). Overall structure of vehicle parking deviation angle detection system.

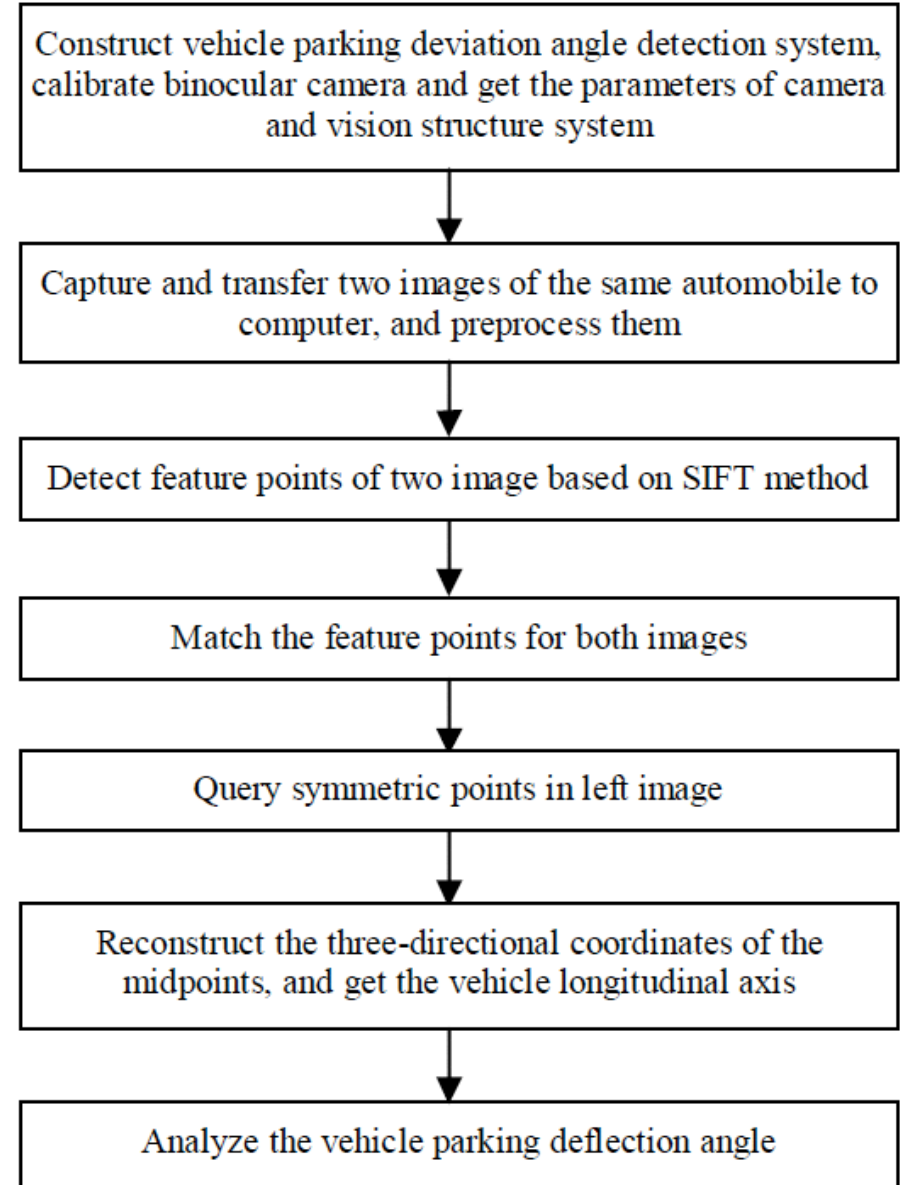

Fig. (3). Realizing process of vehicle parking deviation angle system.

The system mainly consists of the tested automobile, binocular camera and computer. The automobile is parked in the detecting position and the camera is set in front of it. The binocular vision measurement unit is composed of two cameras. The system uses the principle of binocular stereo vision to process the two images of vehicle body, extracts the symmetric points, reconstructs the three-dimensional coordinates and ultimately gets the vehicle parking deviation angle.

\subsection{Process of System Implementation}

This study is started from the calibration of machine vision system, the automobile vision images are processed and the vehicle longitudinal axis is extracted, on this basis of them the vehicle parking deviation angle is acquired. The realization process of computing the vehicle parking deviation angle is shown in Fig. (3).

\section{EXTRACTION OF SYMMETRIC POINTS OF VE- HICLE BODY}

Symmetric point detection is the key step of system implementation where realizing process has four phases. They are image preprocessing, image feature point detection, feature points matching and symmetric point extraction.

\subsection{Extraction of Feature Points for Two Vehicle Images}

Feature point extraction is the basic technology in image processing and computer vision. Usually it is to use the point 
features to discover the corresponding locations in two images and calibrate the camera's parameters [22].

There are mainly three kinds of methods to detect image feature points, algorithm based on gray-scale image, algorithm based on binary image and algorithm based on contour curve [23]. The SIFT algorithm was proposed by Lowe [24, 25] which used the properties of scale space to detect the feature points. It mainly makes use of the information of target local area to construct feature quantity, making the points with extreme values at both scale domain and space domain as the key points and making these key points as the centers of the feature regions. At the same time, the scale of key point can be used to determine the size of feature region. On the basis of histogram of gradient direction in each feature region, local invariant feature vectors can be obtained.

In this study, considering the subsequent symmetric feature matching, the features of gradient orientations and rotational invariance of SIFT are helpful. The feature points in automobile images are extracted based on SIFT algorithm. The process of detecting feature points by using SIFT algorithm is divided into four steps [26].

(1) Extreme point detection in scale space

The potential feature points can be detected by using Gaussian difference formula based on searching all positions and scales of the image.

(2) Key points positioning

The determination of scale and location of every point should be ascertained. At the same time, it is necessary to remote the key points with low contrast and the points in unstable edges. The stability of points matching can be enhanced and the noise immunity can be improved.

(3) Determination of the direction of key points

A direction is assigned for every point, and the all subsequent operations on the image are converted to this direction, scale and location of feature points. The algorithm is invariance to rotation.

\section{(4) Generation of feature point descriptor}

The description vector of feature point is generated by the gradient statistics of surrounding areas of the current scale for key point.

\subsubsection{Construction of Scale Space}

Constructing the scale space is to detect the position that is invariant for the changes of image scale. The scale space of a certain image can be expressed as shown in Eq. 1 .

$$
L(x, y, \sigma)=I(x, y) * G(x, y, \sigma)
$$

Where, $I(x, y)$ is an input image, $G(x, y, \sigma)$ is a Gaussian function with variable scale, and $\sigma$ represents the factor of scale space.

DOG (Difference of Gaussian) scale space is presented to check stable key points effectively in scale space. The space is produced through convolution between different scale of DOG kernel and image [27] as shown in Eq. 2.
$D(x, y, \sigma)=I(x, y) *[G(x, y, a \sigma)-G(x, y, \sigma)]$
$D(x, y, \sigma)=L(x, y, a \sigma)-L(x, y, \sigma)$ bled.

Where, $a$ expresses a factor which is invariable and dou-

It is need to compare each sample point with its all adjacent points in both image domains and scale domain. Then the extreme points in scale space can be found.

\subsubsection{Position Determination for Feature Points}

Once the candidates for feature points are gotten the position, scale, curvature and other information of the stable points will be identified and the candidates that are lowcontrast or in the edges must be filtered [28].

In order to accurately obtain the scale and location of a key point, a quadratic function with three dimensions can be used. The fitting function is shown in Eq. 3.

$D(x)=\frac{1}{2} x^{T} \frac{\partial^{2} D}{\partial x^{2}} x+\frac{\partial D^{T}}{\partial x} x+D$

Where, $x=(x, y, \sigma)^{T}$, the offset relative to the sample point, letting its derivative equation be equal to zero to get the extreme points. Corresponding to the extreme points, the value of equation is shown in Eq. 4.

$\hat{D}(\hat{x})=D+\frac{1}{2} \frac{\partial D^{T}}{\partial x} \hat{x}$

Usually if $|D(x)|<0.03$, the extreme point can be regarded as low-contrast instable feature point which should be removed. At the same time, the exact location and scales of the feature point are also obtained.

If the DOG is defined not well, the principal curvature of extreme value is larger in edge intersection, while it is smaller in the direction perpendicular to edge. The principal curvature is analyzed through a Hessian matrix $H$, assuming its eigenvalues are $\alpha$ and $\beta$. Supposing that $\alpha$ is greater and $\beta$ is smaller so that there is a relation as shown in Eq. 5.

$\frac{\operatorname{Trac}(H)^{2}}{\operatorname{Dete}(H)}=\frac{(\alpha+\beta)^{2}}{\alpha \beta}=\frac{(\gamma \beta+\beta)^{2}}{\gamma \beta^{2}}=\frac{(\gamma+1)^{2}}{\gamma}$

Where, $\operatorname{Dete}(H)$ indicates the value of matrix determinant, $\operatorname{Trac}(H)$ represents the trace of matrix. When the two eigenvalues are equal, $(\gamma+1)^{2} / \gamma$ gets the minimum value. To test whether the curvature is small enough or not, the relation is judged as shown is Eq. 6 .

$\frac{(\gamma+1)^{2}}{\gamma}>\frac{\operatorname{Trac}(H)^{2}}{\operatorname{Dete}(H)}$

Where, the value of $\gamma$ changes between 6 and 10 [29].

\subsubsection{Direction Determination of Feature Points}

In order to make the descriptor rotation invariant, each key point needs be assigned one direction by using the local characteristics of image [30]. The gradient magnitude $\operatorname{gm}(x, y)$ 


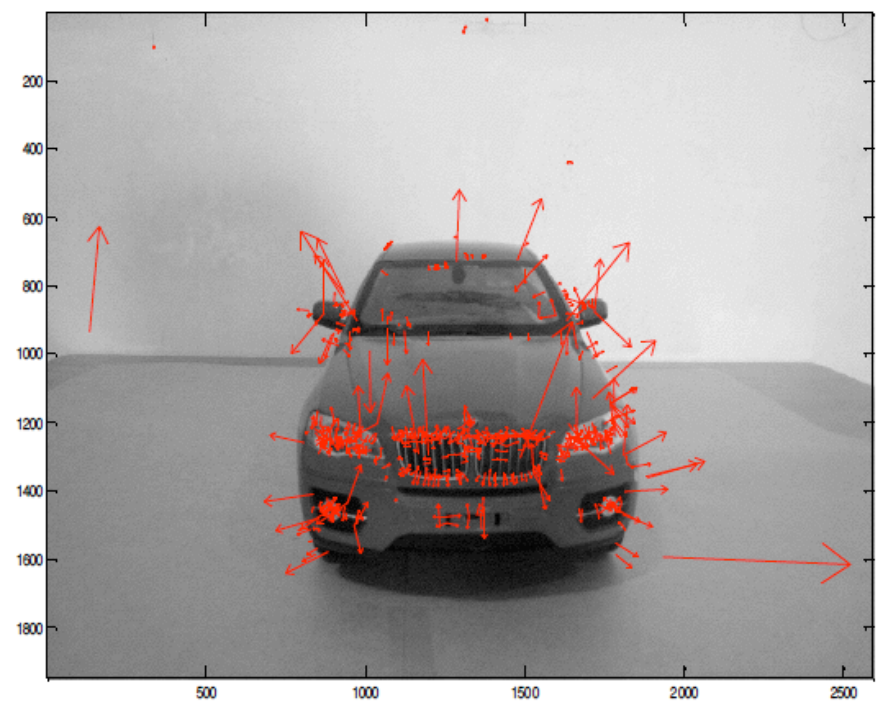

(a) Left image

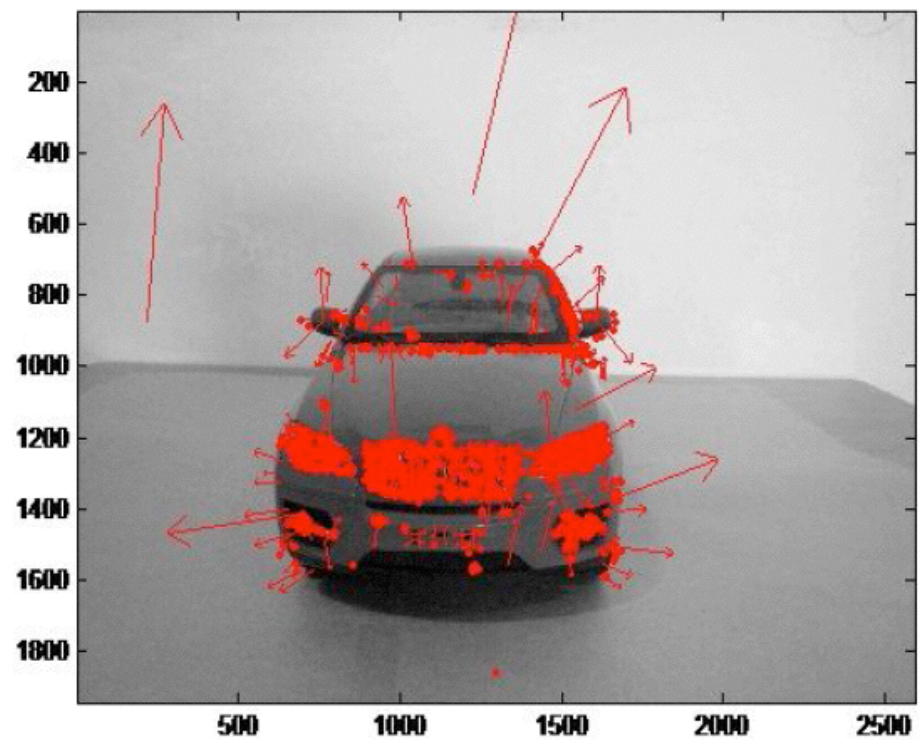

(b) Right image

Fig. (4). Detected feature points of two images by SIFT method.

and gradient orientation $\omega(x, y)$ can be gotten [31] as shown in Eq. 7.

$$
\begin{aligned}
& g m(x, y)=\sqrt{[L(x, y+1)-L(x, y-1)]^{2}+[L(x+1, y)-L(x-1, y)]^{2}} \\
& \omega(x, y)=\arctan \frac{L(x, y+1)-L(x, y-1)}{L(x+1, y)-L(x-1, y)}
\end{aligned}
$$

According to the gradient orientations in certain fields, it is possible to compute the histogram of orientation. If there is a peak in histogram, it means that the neighborhood gradient of key point has a primary orientation [32]. When a peak is equal to four-fifths of the major peak, the direction is believed as the auxiliary direction [33].

\subsubsection{Determination of Feature Point Descriptor}

The descriptor should be different with others so as to facilitate feature points matching. Rotating the coordinate axis to orientation of the key point ensures that it is invariant in rotation. For each key point 16 seed points, each with $8 \mathrm{vec}-$ tors are used to describe it. So each key point generates 128 data, that is, the 128-dimensional SIFT feature vectors are finally created [34]. Then the robustness of matching can be enhanced.

By the SIFT method, the detected feature points of two images of a car model are shown in Fig. (4). There are 639 and 1886 features points in the two images respectively.

\subsection{Feature Points Matching}

It is a basic problem to determine the correspondences of feature points in two images, which is applied widely in image processing and machine vision [35]. Generally, it has two steps: similarity measurement of feature points and mismatching eliminating. 


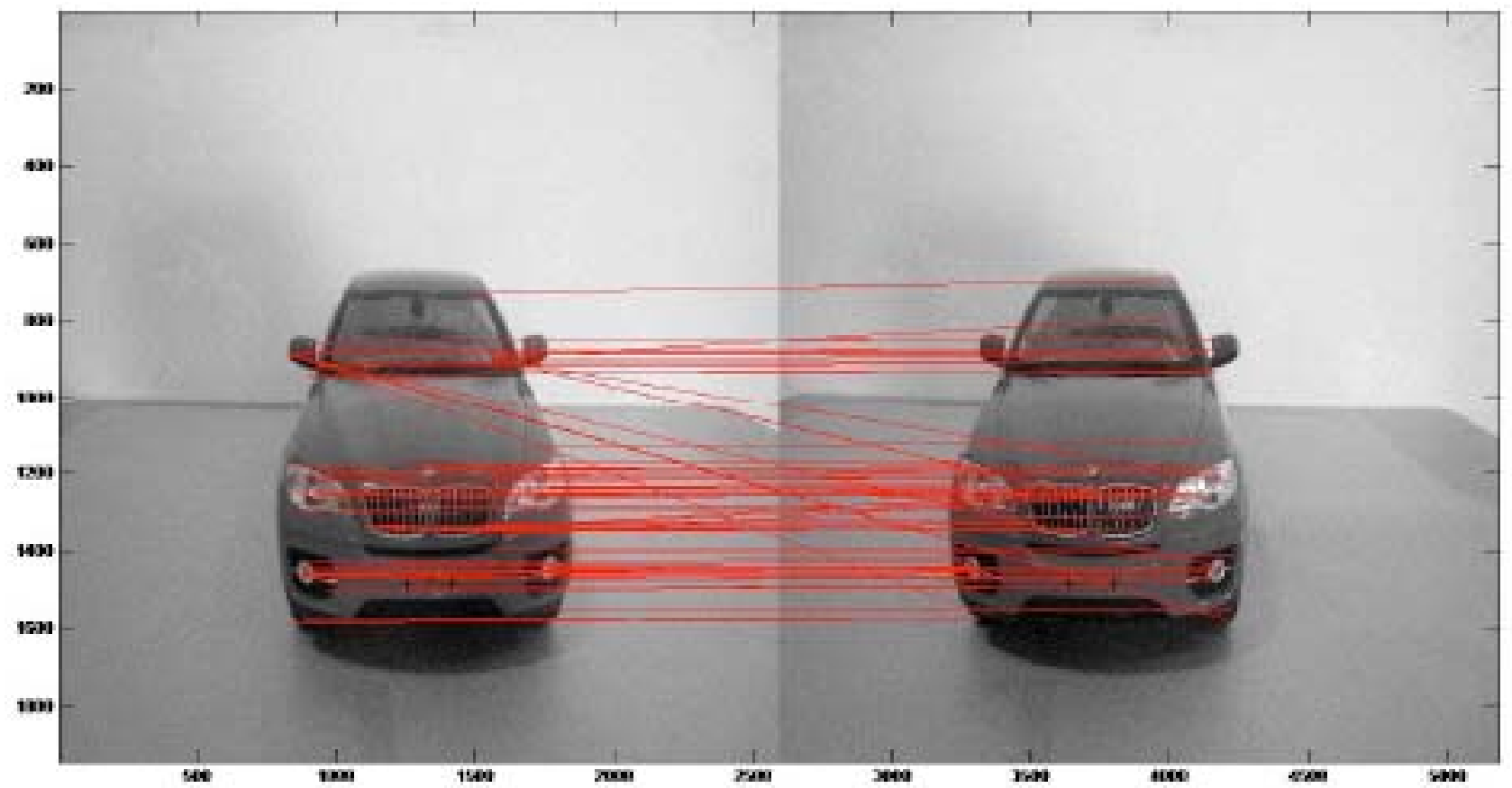

Fig. (5). The feature point matching results based on bidirectional searching Euclidean distance method.

\subsubsection{Similarity Measurement of Feature Points}

After the feature points are detected, those in two images need be matched assuming that the two corresponding feature point sets are given as shown in Eq. 8.

$$
\begin{aligned}
& F_{l}=\left\{f_{1}^{(\text {left })}, f_{2}^{(\text {left })}, \ldots f_{L_{m}}^{(\text {left })}\right\} \\
& F_{r}=\left\{f_{1}^{(\text {right })}, f_{2}^{(\text {right })}, \ldots f_{R_{m}}^{(\text {right })}\right\}
\end{aligned}
$$

Where, $L_{m}$ is the point numbers in left image, and $R_{m}$ is that in right image.

Considering that the Euclidean distance is mature, it is used to measure the features similarity. When SIFT feature vector is n-dimensional the Euclidean distance is shown in Eq. 9.

$d\left(F_{l}, F_{r}\right)=\sqrt{\sum_{i=1}^{n}\left(f_{i}^{(l e f t)}-f_{i}^{(r i g h t)}\right)^{2}}$

Feature points are matched according to distance ratio criteria, that is, for a certain feature point, supposing that the nearest and second nearest Euclidean distances between this point and points in other image are respectively $d_{1}$ and $d_{2}$, the distance ration is defined, as shown in Eq. 10.

$r=\frac{d_{1}}{d_{2}}$

The Feature points matching can be seen successful if it meets the requirement as shown in Eq. 11.

$r<T h$
Where, $T h$ is a certain threshold when the distance ratio is less than the value, matching is successful, on the contrary the feature points matching fails [36].

\subsubsection{Mismatching Eliminating}

The feature point matching algorithm based on Euclidean distance is simple, but the selection of the value of $r$ affects the matching results [37]. Moreover the method can produce overmatching matching particularly when $r$ is larger.

To reduce the mismatching rate the feature points matching algorithm based on bidirectional searching is brought forward on the basis of Euclidean distance, which process is as follows:

(1) An appropriate value of distance ratio is set.

(2) A feature point in left image is selected, which matched feature points are gotten in right image based on the Euclidean distance; and loop forever until all feature points in left image have obtained their matched points in right image. Finally, the feature points matching array of left image LtoR [] is established.

(3) Similarly for the right image, total feature points have acquired their matched points in left image and the matching array RtoL[] of feature points in right image is established.

(4) The two arrays are queried if the feature points $P_{L}$ and $P_{R}$ in two images are matched in the two ways matching, the pair of points $\left(P_{L}, P_{R}\right)$ is considered as a matched point pair. Repeatedly, all matched points can be gotten.

In this study let $T h=0.8$, the number of matched pairs is 97 from left to right and that is 243 from right to left and the number of bidirectional matching is 81 . The final feature point matching results are shown in Fig. (5). 

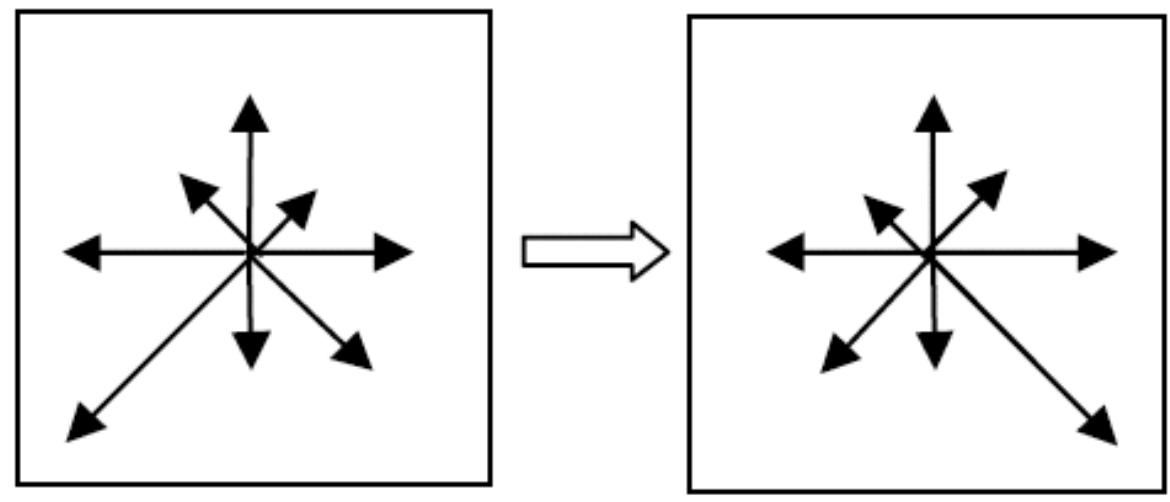

Fig. (6). Symmetric amending of matched feature points of right half in left image.

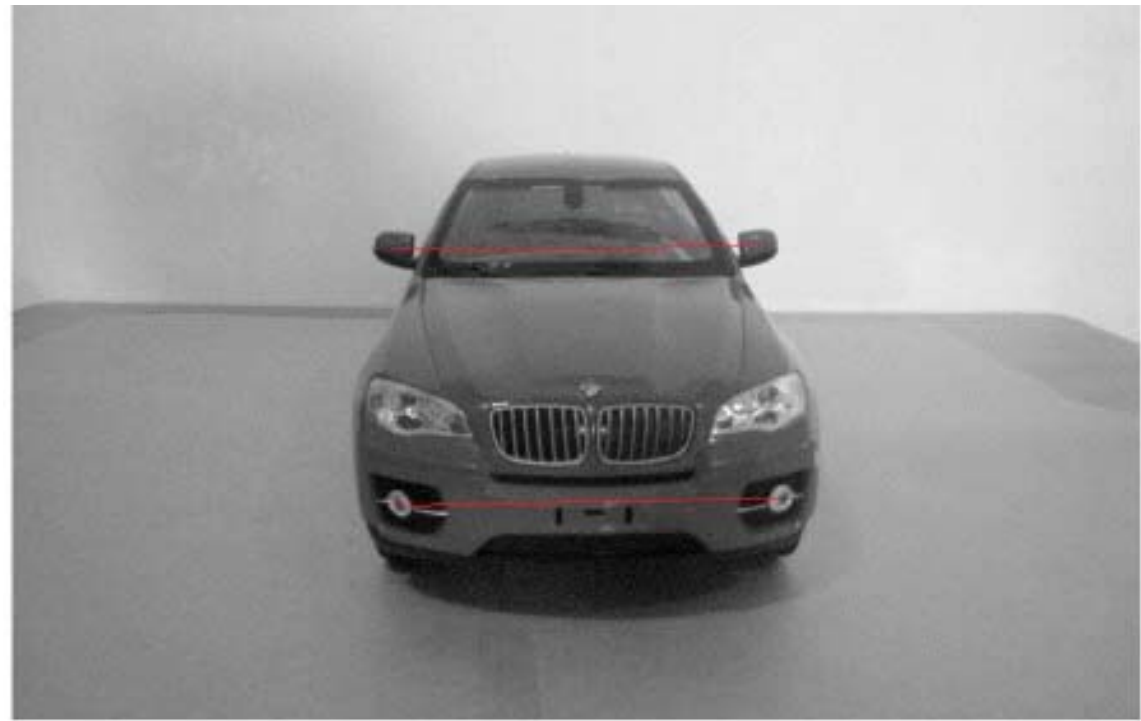

Fig. (7). Detected symmetric pairs of feature points in left image.

\subsection{Detection of Symmetric Points on Vehicle Body}

After the feature points in both images are matched, the feature points in an image left or right should be queried to find symmetric feature points.

The research on symmetry detection of image has yielded some results, the proposed methods include based on statistics [38], based on phase information [39, 40], and so on. However, there are few studies on symmetric point detection.

Because the symmetric point detection of vehicle image studied in this project is similar to feature point matching and the feature point extraction is based on SIFT algorithm, the vehicle is typically object of axial symmetry. The detected feature points can be divided into two parts according to the symmetric axis, in the ideal case the feature points are approximately distributed symmetrically along the center axis of image.

To detect the symmetric points from the matched feature points in left image, they are divided into two parts: feature point set of left half of image $F_{l l}=\left\{f_{1}^{(l o l)}, f_{2}^{(l o l)}, \ldots f_{L_{l m}}^{(l o l)}\right\}$ and point set of right half $F_{l r}=\left\{f_{1}^{(r o l}, f_{2}^{(r o l)}, \ldots f_{L_{m}}^{(r o l)}\right\}$, where, $L_{l m}$ and
$L_{r m}$ are respectively the number of matched points of left half plane and right half plane.

Assuming that a feature point is selected from $F_{l l}$, one of which seeds is $(l s, l t)$ and another feature point is selected from $F_{l r}$, one of which seeds is $(r s, r t)$ where the sets of value of $l s, l t$ and $r s, r t$ are $(1,2,3,4)$. According to the symmetric feature, in the ideal case the subscripts of seed point should have symmetric relation as shown in Eq. 12.

$r s=l s, r t=5-l t$

Besides, the orientation vectors should have the symmetric relation as shown in Fig. (6).

According to the above symmetric relations, the all feature vectors of $F_{l r}$ are amended symmetrically. The symmetric point detection of vehicle body in left image is realized by feature point matching method based on the Euclidean distance.

The duplicate symmetric points are removed to decrease the rate of error querying. Finally, two pairs of symmetric feature points of vehicle body in left image are obtained as shown in Fig. (7). 


\section{DETECTION OF VEHICLE PARKING DEVIATION ANGLE}

On the basis of detected symmetric points, in order to obtain vehicle parking deflection angle using three dimensional reconstructing can get the coordinates of symmetric points and analyze the longitudinal axis.

\subsection{Calibration of Binocular Stereo Vision System}

The Camera can transform the object space to image plane [41]. In the binocular stereo vision system, threedimensional information can be obtained from two dimensional images. The relations between positions are determined by camera parameters, they can be found by camera calibration.

The technology is a very important aspect for machine vision. It determines the accuracy of measurement or threedimensional reconstruction in machine vision application system [42].

Because the production of two-dimensional target is convenient and cheap, the camera calibration algorithm based on two-dimensional target has been widely applied. In this study, the camera is calibrated by Zhang plane method, which is realized based on planar grid points target [43, 44].

The system calibration of binocular stereo vision means that when the internal parameters of left camera and right camera are calibrated, the vision system structural parameters can be determined mainly including translation vector and rotation matrix, or $T$ and $R$. For the both images the coordinates of corresponding feature points are normalized, supposing that the ideal coordinates are respectively $\left(X_{l}, Y_{l}\right)$ and $\left(X_{r}, Y_{r}\right)$.

For the left camera, the external parameters are $R_{l}$ and $T_{l}$ and the ones for right camera are $R_{r}$ and $T_{r}$. For any point, assuming that in left, right camera coordinate systems and world coordinate system, the non-homogeneous coordinates are respectively $x_{l}, x_{r}$ and $x_{w}$. Then, they have a relation as shown in Eq. 13.

$x_{l}=T_{l}+R_{l} x_{w}$

$x_{r}=T_{r}+R_{r} x_{w}$

The geometric relation between the two cameras can be expressed as shown in Eq. 14.

$$
\begin{aligned}
& R=R_{r} R_{l}^{-1}, \\
& T=T_{r}-R_{r} R_{l}^{-1} T_{l}
\end{aligned}
$$

In this project, the two cameras simultaneously are calibrated by the same calibration target to get their intrinsic and external parameters. At the same time, the system structure of binocular stereo vision are calibrated which results of $R$ and $T$ are as follows:

$$
R=\left[\begin{array}{ccc}
0.9999 & -0.0065 & 0.0128 \\
0.0065 & 1.0000 & -0.0040 \\
-0.0127 & 0.0041 & 0.9999
\end{array}\right]
$$

$T=[-35.0324,0.2711,-3.4069]$

\subsection{Analysis of Vehicle Parking Deviation Angle}

According to the analyzing results of the symmetric points in left vehicle image, the coordinates of two pairs of symmetric points on both images are gotten. Then the coordinates of midpoints of lines connecting the symmetric feature points pairs in both images are also obtained, the measurement data of the experiment are shown in Table $\mathbf{1 .}$

Table 1. Feature points coordinates of symmetric point pairs in both images.

\begin{tabular}{|c|c|c|c|c|c|}
\hline \multicolumn{2}{|c|}{ Feature Point } & \multicolumn{2}{c|}{ Left Image } & \multicolumn{2}{c|}{ Right Image } \\
\hline \hline \multirow{2}{*}{$\begin{array}{c}\text { Symmetric } \\
\text { point } \\
\text { pair } \\
1\end{array}$} & Point 1 & 872.47 & 881.00 & 762.57 & 874.30 \\
\cline { 2 - 6 } & Point 2 & 1720.32 & 875.75 & 1611.13 & 874.15 \\
\cline { 2 - 6 } & midpoint & 1296.40 & 878.38 & 1186.85 & 874.23 \\
\hline \multirow{2}{*}{$\begin{array}{c}\text { Symmetric } \\
\text { point } \\
\text { pair } \\
2\end{array}$} & Point 1 & 889.55 & 1458.60 & 697.57 & 1444.46 \\
\cline { 2 - 6 } & Point 2 & 1772.48 & 1440.99 & 1583.46 & 1447.78 \\
\cline { 2 - 6 } & midpoint & 1331.02 & 1449.80 & 1140.52 & 1446.12 \\
\hline
\end{tabular}

According to the principle of binocular vision for the left camera coordinate system, the coordinates of the two midpoints can be computed, they respectively are $(11.15,-19.76$, 424.66) and (12.37, 64.92, 292.36).

Since the left camera coordinate system is inconsistent with the testing site in this study, and then the two coordinates are translated into the coordinates in the world coordinate system, which are $(135.05,145.65,192.92)$ and $(189.27,150.44,340.27)$. Then, the vector of vehicle longitudinal axis is $(54.22,4.79,147.35)$. Finally the vehicle parking deviation angle can be obtained, which value is $1.4223^{\circ}$ and the deviation is to right.

In order to verify the correctness of the detection scheme, this study conducts a number of experiments and field measurement, the actual field measurement data is $1.3902^{\circ}$. The experimental results of the detecting system are shown in Table 2. The absolute value of the maximum error is less than $0.15^{\circ}$ which can meet the requirement of the detection system design.

\section{CONCLUSION}

On the basis of several methods of analyzing vehicle parking deviation angle, which advantages and disadvantages are summarized and compared. An approach of computing the deviation angle based on binocular stereo vision is proposed through the technologies of SIFT feature point detection and matching.

The result of experiment in this study suggests that, according to SIFT feature points extraction and description the bidirectional matching method can match the feature points 
Table 2. The measurement results of seven experiments and the field measurement data.

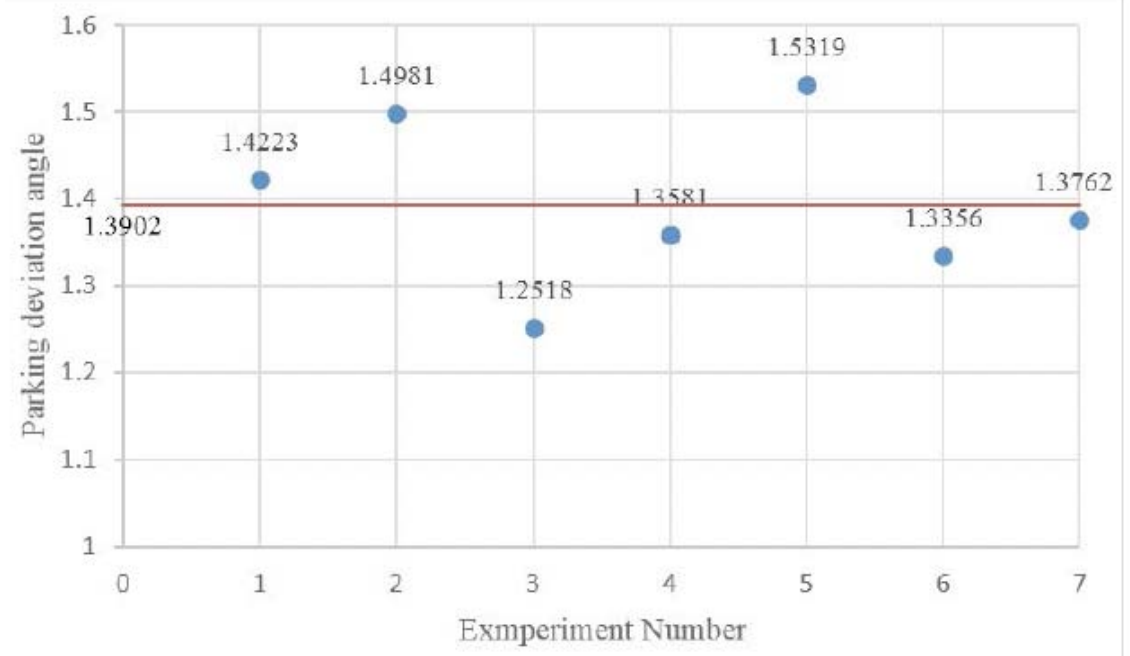

in left and right vehicle images effectively. The bidirectional searching method based on Euclidean distance can reduce the error matching rate. The extraction method of symmetric points based on feature symmetry amending can query the symmetric points in left image. Based on the calibration of binocular stereo camera, by taking three-dimensional reconstruction the midpoints of lines connecting symmetric points can be used to get the coordinates in the world coordinate system which is accordance with the testing field. Finally, the longitudinal axis of vehicle is extracted and the vehicle parking deviation angle is received. The method is noncontact measurement, and it has good real-time performance and high automation degree. It can lay good foundation for the subsequent vehicle detection application and it has certain theoretical and practical value.

Through the process of realizing vehicle parking deviation angle, the measurement precision and accuracy of this study mainly depend on the technologies of feature point detection, feature point matching algorithms and symmetric point detection algorithm. The limitation of this method is that the number of detected symmetric points is limited, in this case, there are only two pairs of symmetric points, it is clear that the number is fewer. Further more, the feature points matching and symmetric feature points querying sometimes go wrong then the measurement results may have large errors. Therefore to improve the detection accuracy and the practicality of vehicle parking deviation angle, the more accurate feature point detection and matching algorithms and practical symmetric feature points querying algorithm are the focuses in the future research.

\section{CONFLICT OF INTEREST}

The authors confirm that this article content has no conflicts of interest.

\section{ACKNOWLEDGEMENTS}

This study is supported by the Graduate Student Research and Innovation Program of Jiangsu Province (No. CXLX13_513) and the Science and Technology Devel- opment Planning Project of Shandong Province (No. 2013 YD05004), P. R. China.

\section{REFERENCES}

[1] R. Taormina, K.W. Chau and R. Sethi, "Artificial neural network simulation of hourly groundwater levels in a coastal aquifer system of the Venice lagoon," Engineering Applications of Artificial Intelligence, vol. 25, no. 8, pp. 1670-1676, 2012.

[2] C. Cheng, K.W. Chau, Y. Sun and J. Lin, "Long-term prediction of discharges in Manwan Reservoir using artificial neural network models," Lecture Notes in Computer Science, vol. 3498, pp. 1040$1045,2005$.

[3] K.W. Chau, "Application of a PSO-based neural network in analysis of outcomes of construction claims," Automation in Construction, vol. 16, no. 5, pp. 642-646, 2007.

[4] J. Zhang and K.W. Chau, "Multilayer ensemble pruning via novel multi-sub-swarm particle swarm optimization," Journal of Universal Computer Science, vol. 15, no. 4, pp. 840-858, 2009.

[5] C.L. Wu, K.W. Chau and Y.S. Li, "Predicting monthly streamflow using data-driven models coupled with data-preprocessing techniques," Water Resources Research, vol. 45, no. 8, pp. W08432, 2009.

[6] L.J. Zhu, "Research on the test technology of automobile headlamp based on image processing," Master Thesis, Nanjing Forestry University, Nanjing, China, 2005.

[7] H.Y. Zhu, "Research on the image processing technology for automobile headlamp testing," Master Thesis, Nanjing Forestry University, Nanjing, China, 2007.

[8] Y.J. Min, L.J. Zhu, H.Y. Zhu and Z.J.Xu, "Design and implementation of correction system for measuring error of automobile headlamp beam," Journal of Nanjing Forestry University (Natural Science Edition), vol. 32, no. 2, pp. 107-110, 2010.

[9] Z.K. Huang and K.W. Chau, "A new image thresholding method based on Gaussian mixture model," Applied Mathematics and Computation, vol. 205, no. 2, pp. 899-907, 2008.

[10] Y. Yun, S. Dai, P. Mu, and L. Wang, "Study on direction detection of automobile headlamp beam," in 4th International Symposium on Advanced Optical Manufacturing and Testing Technologies: Optical Test and Measurement Technology and Equipment, pp. 72832B-72832B, 2009.

[11] S.Y. Wang, "Study on the form of deviation of the automobile bodywork examination instrument," Master Thesis, Jilin University, Changchun, China, 2005.

[12] P.C. Chen and Y.J. Min, "Study on placement correction method of automobile headlamp detection based on camera calibration," Journal of Applied Sciences, vol. 13, no. 4, pp. 525-535, 2013.

[13] H.Y. Lin, "Study on testing system of the position deviation of vehicle-body and axle based on stereo vision," $P h$. D. Thesis, Jilin University, Changchun, China, 2008. 
[14] P.D. Wu, "Study on correction method of parking error in automobile headlamp testing," Master Thesis, Changchun, China, 2009.

[15] J.G. Hou, "Research on modelling and simuation of the parkaing car axle slant angle based on the tire outline identification," Master Thesis, Jilin University, Changchun, China, 2009.

[16] L. Zhang, S. Ge, H. Shan, Y. Tian, and J. Hou, "Research on detecting and simulating of deflective angle of axle placed," in International Conference on Intelligent Computation Technology and Automation, pp. 1142-1145, 2010.

[17] L. Zhang, S. Jian, G. Shubin, L. Yicai, and H. Jiguang, "Error correction device of headlamp detection and experimental analysis," in 2nd International Conference on Signal Processing Systems, pp. V1411-V1415, 2010.

[18] H. Shan, S. Lili, P. Songyan, G. Shubin, and Z. Libin, "Simulation analysis of error correction method of headlamp detection based on driving direction," in IEEE International Conference on Wireless Communications, Networking and Information Security, pp. 290294,2010

[19] S.D. Ma and Z.Z. Zhang, Computer Vision, Beijing: Science Press, 1998.

[20] L. Yan, "3D stereoscopic imaging: An overview and speculation on its engineering applications," Development \& Ommpvatoin of Machinery \& Electrical, no. 4, pp. 32-34, 2010

[21] G. J. Zhang, Machine Vision, Beijing, China: Science Press, 2005.

[22] R. Szeliski, Computer Vision: Algorithms and Application, Springer, 2010.

[23] Y. Ren, "A survey of corner detection algorithms," Mechanical Engineering \& Automation, no. 1, pp. 198-200, 2009.

[24] D.G. Lowe, "Object recognition from local scale-invariant features," in The proceedings of the seventh IEEE international conference on Computer vision, Kerkyra, pp. 1150-1157, 1999.

[25] D.G. Lowe, "Distinctive image features from scale-invariant keypoints," International journal of computer vision, vol. 60, no. 2, pp. 91-110, 2004.

[26] D.Z. Cheng, Y.J. Li, and R.X. Yu, "Image matching method based on improved SIFT algorithm," Computer Simulation, no. 7, pp. 285-289, 2011.

[27] L. Juan and O. Gwun, "A comparison of sift, pca-sift and surf," International Journal of Image Processing, vol. 3, no. 4, pp. 143$152,2009$.

[28] J. Feng, "The research and improvement of SIFT Altorithm," Master Thesis, Jilin University, Changchun, China, 2010.

[29] L.L. Yu and Q. Dai, "Improved SIFT feature matching algorithm," Computer Engineering, no. 2, pp. 210-212, 2011.
[30] Z. A. Pan, "Research on image mtching based on SIFT altorithm," Master Thesis, Xidian University, Xi' an, China, 2012.

[31] C.-Y. Tang, Y.-L. Wu, M.-K. Hor, and W.-H. Wang, "Modified sift descriptor for image matching under interference," in International Conference on Machine Learning and Cybernetics, Kunming, China, pp. 3294-3300, 2008.

[32] W.G. Song, H.X. Guo, and Y. Wang, "A method of fruits recognition based on SIFT characteristics matching," in IEEE International Conference on Artificial Intelligence and Computational Intelligence, pp.119-122, 2009.

[33] J. Xie, B.H Li, W. Han, J. HBao, F. Gu, and F. Guo, "A tiny facet primitive remote sensing image registration method based on SIFT key points," in International Symposium on Instrumentation \& Measurement, Sensor Network and Automation, pp. 138-141, 2012.

[34] C.M. Zhang, Z.H. Gong, and L. Sun, "Improved SIFT feature applied in image matching," Computer Engineering and Applications, no. 2, pp. 95-97, 2008.

[35] E. Delponte, F. Isgrò, F. Odone, and A. Verri, "SVD-matching using SIFT features," Graphical Models, vol. 68, no. 5-6, pp. 415431, 2006.

[36] J.Z. Guo, "License plate identification based on SIFT algorithm," Master Thesis, Xidian University, Xi' an, China, 2012.

[37] S. Qian and J.Y. Zhu, "Improved SIFT-based bidirectional image matching algorithm," Mechanical Science and Technology for Aerospace Engineering, vol. 27, no. 9, pp. 1179-1182, 2007.

[38] G. Wu, J.A. Yang, D.L. Li and H. Zhang, "On the methods of symmetry detection of the plane images," Computer Science, vol. 28, no. 5, pp. 101-103,106, 2001.

[39] H.B. Yang, "Symmetry detection in vision description," Master Thesis, Hebei University of Technology, TianJin, China, 2002.

[40] M. Yu, Z.T. Xiao, H.Z. Zhang and Y.C. Guo, "Phase congruency based symmetry detection in image feature description," Journal of Hebei University of Technology, vol. 33, no. 3, pp. 38-41, 2004.

[41] T. Clarke and J. Fryer, "The development of camera calibration methods and models," The Photogrammetric Record, vol. 16, no. 91, pp. 51-66, 1998.

[42] C.C. Li, "Study and realization of camera calibration technology in machine vision," Master Thesis, Nanjing University of Aeronaltics and Astronautics, Nanjing, China, 2009.

[43] Z. Zhang, "A flexible new technique for camera calibration," IEEE Transactions on Pattern Analysis and Machine Intelligence, vol. 22, no. 11, pp. 1330-1334, 2000.

[44] Z. Zhang, "Flexible camera calibration by viewing a plane from unknown orientations," in The Proceedings of the Seventh IEEE International Conference on Computer Vision, pp. 666-673, 1999.

(C) Chen and Min; Licensee Bentham Open

This is an open access article licensed under the terms of the Creative Commons Attribution Non-Commercial License (http://creativecommons.org/licenses/by-nc/3.0/) which permits unrestricted, non-commercial use, distribution and reproduction in any medium, provided the work is properly cited. 